\title{
TÓPICOS SOBRE ADMINISTRACIÓN Y EVALUACIÓN: UNA CONVERSACIÓN CON LAS IDEAS DE MAURICIO TRAGTENBERG*
}

\section{Agueda Bernardete Bittencourt Uhle}

Escribir puede ser un duro oficio, pero algunas veces llega a ser mágico. En este momento no tengo un tema panicular sobre el cual deba detenerme, pensar, desglosar. Quiero que este texto esté orientado por los recuerdos y por el sentimiento, materiales con los cuales pretendo alivianar las ideas. El sentimiento que hoy experimento es difícil de precisar, yo lo llamaría de desamparo. No sé, incluso si desamparo puede ser un sentimiento o una sensación, pero el lector sabrá de lo que hablo.

Desamparo remite por oposición a una figura fuerte, acogedora, al calor de la solidaridad, a la sensación de bienestar de tener un hombro amigo en quien apoyarse. El desamparo a que me refiero tiene que ver con la ausencia de un buen consejero, dispuesto a escuchar, a discutir y a buscar juntos el mejor camino. Ausencia de aquel que sabe hacer la pregunta clave sobre los asuntos más cotidianos. Entiendo mejor ahora la música que Sergio Bittencourt hizo en homenaje a Jacó do Bandolim y que dice: "En aquella mesa está faltando él y la nostalgia de él está doliendo en mí".

No poder encontrar más a Mauricio y discutir con él el rumbo de los Bancos, la negociación de la Ford con el gobierno de Río Grande del Sur, la posible candidatura de fulano o sutano a la presidencia de la República o las implicaciones de las acciones de la OTAN en Kosovo, deja una sensación de desamparo. Pero el desamparo, el vacío de esa ausencia se vuelve todavía mayor cuando los problemas políticos y sociales están disfrazados, o enmascarados, difícilmente identificados como temas políticos de tan largo alcance como los anteriormente citados, de evidente visibilidad.

Aquí me refiero a la falta que me hacen las conversaciones a la hora del almuerzo, cuando hablábamos de la vida cotidiana y surgían temas como las relaciones entre hombres y mujeres, en la casa, en el trabajo, las marcas de la cultura judeo-cristiana que cada uno de nosotros trae en su forma de mirar el mundo, de los símbolos que tocan a unos y a otros de acuerdo con sus historias personales. Sin esnobismo alguno, con la simplicidad que lo caracterizó, Mauricio traía ejemplos históricos que inmediatamente me hacían sentir un personaje de mi tiempo, coherente con la historia y con el lugar donde estoy. Esa posición tranquila que él siempre adoptaba, que sólo garantiza el conocimiento, transmitía seguridad, confianza, daba ligereza a los temas más complejos.

\footnotetext{
* Texto elaborado con ocasión del Seminario sobre "Mauricio Tragtenberg: "la búsqueda de la coherencia entre el pensar y el hacer" realizado en la Universidad Estadual de Londrina, entre el 14 y 16 de junio de 1999. Traducción de Martha Cecilia Herrera y Guillermo Bustamante Z, profesores de la Universidad Pedagógica Nacional.

Profesora de la Universidad de Campinas, SP, Brasil. Investigadora grupo FOCUS, Departamento de Administración Educativa, Facultad de Educación.
} 
La erudición era parte integrante de su espíritu, fluía naturalmente. Era capaz de hacer indicaciones bibliográficas a los pos-doctorados y PHDs, con la misma gentileza y naturalidad con que indicaba lecturas a los alumnos de pregrado que lo abordaban en los corredores y cafeterías de la universidad.

Me podría quedar hablando durante horas y horas para demostrar la legitimidad de mi desamparo, pero no es de eso que trata este seminario. Escogí, pues, del conjunto de la obra de Mauricio, aquel texto que está más fuerte en mis recuerdos y que tiene mucho que decir en el debate que se establece hoy sobre Educación, Política Universitaria y Estado, y que fue mi referencia en la práctica docente por varios años.

Me gustaría contar cómo ese texto me enseñó a leer la historia contemporánea, a comprender los procesos políticos que en plena década de los 70 eran considerados técnicos. Me hizo entender que la administración, en lugar de una actividad técnica, es un claro ejercicio político. Se trata del uso del poder. Me refiero al libro Burocracia e Ideologia ${ }^{1}$.

Pero, ante todo, juntando desamparo y remembranzas recuerdo la escena de mi primer encuentro con el profesor Tragtenberg, que tiene que ver con el tema del libro en cuestión. Era una sala en el edificio del ciclo básico, en la Universidad de Campinas, UNICAMP, una sala tipo auditorio, enorme y llena. Agosto de 1977, primera clase de Administración Educativa, en el programa de la maestría. Entro en la sala a las 2:30 de la tarde, con media hora de atraso; encuentro el tablero lleno de anotaciones desorganizadas y sobre el estrado profesoral, un señor que hablaba con un cigarrillo en la boca, con voz calmada y pausada. Cuando me vio entrar, interrumpió su exposición para recibirme a su modo: "Entonces, doña María, ¿se atrasó lavando la loza? Es bueno llegar a tiempo si no pierde la materia y le va mal en el examen...". Esta mezcla de ironía y crueldad afectó directamente mi feminidad. Yo tenía mis razones para llegar atrasada ese día. Luego descubrí que él también tenía las suyas para no soportar que se abriera la puerta después que él iniciara la clase. Cosas de la historia de cada quien. Yo con mi maternidad y él con las marcas de la persecución política y delación que había sufrido en los años anteriores. En el tablero, en medio de las anotaciones en desorden, conseguí descifrar tres referencias que me han sido útiles hasta hoy, 22 años después: Nicolás Maquiavelo, El Príncipe; Franz Kafka, El Proceso; Max Weber, Economía y Sociedad. Había más, pero yo sólo conseguí llegar a esas tres, de las cuales saqué más tarde las bases para mis propias reflexiones sobre administración educativa. Muchas otras vinieron después; éstas, sin embargo, permanecen en mi horizonte en la actualidad, cuando estoy dedicada a estudiar lo laico y lo religioso en la educación, asunto tratado de forma diferente por cada uno de estos autores.

En este mismo año de 1977, Mauricio lanzaba su libro Burocracia e Ideo/ogía a cuya lectura me dediqué un año después. Mi primera reacción fue de desánimo. Era muy denso, yo no tenía referencias para entender lo que allí estaba siendo analizado; fue entonces que discutiendo o, mejor, quejándome al autor, que aprendí para el resto de mi vida. Mauricio me dijo simplemente lo siguiente: "Me tomó 6 años leer Economía y Sociedad de Weber". Leer un libro no es solamente seguir las frases escritas por el autor, es preciso comprender las bases del pensamiento que está expuesto, saber con quién el autor está discutiendo, de quién es heredero. En síntesis, la producción de un autor está situada en la historia, en el universo del pensamiento sobre el tema que escribe. Lo que Mauricio me dijo ese día fue que

\footnotetext{
${ }^{1}$ Tragtenberg, Mauricio. Burocracia e Ideologia. Sao Paulo: Editora Ática, 1977. Este libro fue originalmente la tesis de doctorado defendida por Mauricio en la Universidad de Sao Paulo. 
no entendería su libro si no iba a los pie de página y no buscaba sus fuentes y sus interlocutores.

¡Valió la lección! Volví al libro y por un semestre me dediqué a la lectura y comprensión del capítulo II, "Las armonías administrativas, de Saint-Simon a Elton Mayo". Con este estudio diseñé el programa de la disciplina "Principios y Métodos de Administración Educativa I,', para el curso de Pedagogía. Fue con este estudio que aprendí el significado del tecnicismo en educación que se estableció en Brasil a lo largo de este siglo. Aprendí lo que significa tratar las teorías de la administración a través de sus principios históricos, filosóficos, económicos y sociológicos. Pues, al hacer esa lectura, me vi obligada a estudiar la historia del capitalismo, la importancia de la revolución industrial, los descubrimientos científicos y tecnológicos, las transformaciones en el orden industrial para la producción de una nueva teoría. O, mejor, para el surgimiento de un nuevo campo de conocimiento, como diría $\mathrm{P}$. Bourdieu.

En lugar de trazar el contexto donde las cosas sucedieron o suceden, como se ha hecho en la mayoría de las tesis sobre educación en el Brasil, donde el tal contexto histórico es un capítulo aparte al comienzo de la tesis y que, en general, no ayuda a explicar el problema de estudio, en las obras de Mauricio, y en las tesis que él orientó, los datos históricos y los principios económicos, filosóficos o sociológicos están integrados en el análisis hecho, en la perspectiva en juego sobre el problema de estudio.

Lo que sucede en el capítulo sobre "Las armonías administrativas" es que, de los análisis sobre las condiciones objetivas del trabajo, con base en la infraestructura tecnológica, Mauricio va tejiendo la red de explotación capitalista en relación con el trabajador en el universo fabril, cotejándola con el pensamiento político-económico-social y filosófico de cada época, a partir del siglo XVIII. El problema central para el autor parece ser el de encontrar el lugar de los intelectuales que producen las teorías sobre la sociedad y descubrir a qué y a quién sirven.

Al escoger ese texto como referencia de esta exposición, lo hice justamente porque veo la actualidad de la preocupación en él expresa. Como veremos más adelante, este texto permite comprender perfectamente el significado de algunas políticas académicas que están siendo adoptadas en las universidades más prestigiosas de este país y de otros de América Latina, presentadas como reformas, procesos de modernización, avances democráticos, cuando en verdad no pasan de viejas propuestas de un nuevo tecnicismo.

En este texto me impresionó particularmente la demostración que Tragtenberg hace del paso de las grandes teorías sobre la sociedad y sobre el gobierno, hacia las teorías de las organizaciones que se van a encargar de cronometrar los movimientos del trabajador al pie de la máquina. Se trata de una ruptura en la historia del pensamiento político que tiene sus bases en la dinámica del proceso de industrialización. Se trata del momento en que se hace radical la transformación en las relaciones sociales en el mundo capitalista. En ese momento, la empresa asume el lugar de la sociedad, en el universo del pensamiento y, por tanto, substituye un campo de investigación por otro. No se trata de saber más cuál es la organización social ideal para que los hombres vivan en armonía —como pensaba Fourier, a finales de siglo- o, cómo debe ser gobernada la sociedad, si por los productores, por los 
nobles o por la iglesia — como le preocupaba a Saint-Simon ${ }^{2}$ —. Se trata más bien de si la empresa asume el lugar de la sociedad, si ella misma es su horizonte y si lo que debe ser pensado son las formas de hacerla crecer y fortalecerse. Es, pues, esta transformación en la concepción de la sociedad y de la política lo que va a permitir el surgimiento de la Administración Científica de Taylor.

Los estudios de este ingeniero, antiguo obrero, sobre el proceso de trabajo, centrado especialmente en el control de tiempos y movimientos, marca el surgimiento de las teorías de las organizaciones. Taylor escribe, basado en las observaciones que realizó en empresas americanas, con miras a garantizar mayor lucro y mayor productividad en la industria. Aunque la introducción de su libro haga referencia a la situación americana y a la necesidad de producir para el enriquecimiento del país y para el bienestar de los trabajadores, la teoría de la administración que se inicia con ese pensamiento, va a ocupar el lugar de la teoría política y de los análisis sociales globales, herederos del humanismo. Allí donde antes estaba el hombre y la sociedad, ahora se encuentra la empresa y la productividad.

Tragtenberg, al analizar el surgimiento de estas teorías, va develando las condiciones sociales que tornaron posible, no sólo la emergencia, sino también la expansión del taylorismo, como forma de organización del trabajo. Señala que solamente en un contexto de desempleo, de bajo índice de sindicalización y organización de los trabajadores, de ausencia de legislación social y de existencia de empresas con gran poder económico y político, fue posible que las empresas implantaran tan amplia y masivamente la organización del trabajo pensada por Taylor y más tarde profundizada por Fayol y Elton Mayo.

La teoría de la administración iniciada con la Administración Científica de Taylor está basada en la jerarquía y control, en el distanciamiento del operario (ejecutor) de los planeadores y evaluadores situados en el Departamento de métodos o en el sector de supervisión. En la teoría de la administración no hay lugar para pensar la sociedad y la historia; ella está centrada en las organizaciones, en la producción, en el producto y en los procesos de trabajo. Son los medios y la forma los que están en cuestión; no cabe la discusión sobre los fines o, mejor, sobre los efectos de la generalización de esta organización taylorista del trabajo sobre la sociedad contemporánea.

Pero la historia sigue su curso y el tiempo hace su trabajo. El taylorismo no fue jamás aceptado por los trabajadores. Las formas de resistencia, ausentismo, huelgas, operación tortuga... significaron presión sobre las empresas y sus gestores y dieron origen a nuevas respuestas del capital, en forma de teorías de la administración. La teoría de las relaciones humanas en el trabajo — desarrollada inicialmente por Elton Mayo y Mary Parker Follet-y los estudios realizados por Jacob Levy Moreno, aparecen como tentativas de dorar la píldora amarga del taylorismo, transformando lo superfluo pero manteniendo lo esencial en las relaciones de trabajo, es decir, la separación del trabajador de las decisiones sobre el trabajo, el lucro y el ritmo de producción, garantizando así los mecanismos de explotación y acumulación.

Desglosando esta historia, Tragtenberg va demostrando la naturaleza del modo de producción capitalista, su forma de organización y gestión, que no se restringe simplemente

\footnotetext{
${ }^{2}$ Tragtenberg, M., Op.Cit., pp. 58 -89.

Digitalizado por RED ACADEMICA
} 
a la producción, sino que acaba por invadir todos los espacios de las relaciones humanas, incluidas la escuela, la iglesia, el club.., allí donde quiera que el individuo actúe.

En la escuela, en los años 60 comenzaron a aparecer cursos de formación para administradores y supervisores educativos. Era la división del trabajo en el modelo industrial que se establecía en la escuela. Supervisión, planeación y evaluación pasaron a ser campos de investigación y formación. En la práctica, ser director o supervisor significaba -como en las empresas- hacer carrera, ganar mejores salarios en relación con los profesores.

A partir de la década del 70, Mauricio Tragtenberg, trabajando en la Facultad de Educación de la Unicamp, en el Departamento de Administración y Supervisión Educativa, sigue escribiendo, haciendo docencia, orientando tesis, en la búsqueda de hacer comprender, a los educadores, las consecuencias producidas por tal organización de la educación y de la escuela ${ }^{3}$. Él percibía que el establecimiento de un campo de investigación y formación es un importante factor legitimador de la práctica. La creación de un espacio académico para formar directores y supervisores da origen a una dinámica perversa, difícil de romper, pues se trata de la legitimación prestada por la ciencia. En este caso, legitima el ejercicio del poder revestido de función técnica y especializada en la práctica de las escuelas y de la Secretaría de Educación. Los profesores, aquellos que permanecieron en el aula, rápidamente percibieron los efectos de burocratización de la escuela, concomitantes a la división social del trabajo. Iniciaron, en la década del 80 , una lucha por la democratización de la escuela y contra la jerarquía burocrática de la Secretaría de Educación, que duró 20 años en el estado de Sáo Paulo.

La extensión y dureza de la lucha se debió al hecho de que una vez alcanzado un cargo o función de dirección, los ex-profesores pasan a defender, con uñas y dientes, los privilegios de estos cargos. Es de ahí que surgen las asociaciones de directores y supervisores, encargados de defender los derechos adquiridos por sus asociados, oponiéndose lógicamente a quienes permanecen en el aula.

Por otro lado, en las universidades, las facultades de educación, que se habían estructurado por departamentos —ofreciendo la formación técnica a los cuadros de la escuela (supervisores, orientadores, inspectores y directores) - luchaban por mantener estos cargos en las escuelas de primaria y secundaria, preservando de esta forma sus propias actividades, sus campos de investigación y áreas de entrenamiento.

Esta posición, que se mantuvo en la Unicamp por lo menos hasta 1997, merece ser mejor analizada. Para las escuelas primaria y secundaria, los profesores universitarios defendían el nombramiento por concurso de directores y supervisores, la estabilidad y la formación técnica; mientras que, para la gestión universitaria, defendían la rotación en los cargos y la elegibilidad de todos los profesores, independientemente del área de formación. En otros términos, "la fórmula ofrecida a los otros no nos sirve a nosotros".

Pero, como bien señalaba Tragtenberg, en concordancia con Hanna Arendt y Bourdieu entre otros, la educación, conservadora por naturaleza, sigue en contravía del proceso histórico. Así, mientras los educadores luchan, unos por el mantenimiento y otros por la

\footnotetext{
${ }^{3}$ Entre los textos básicos sobre el tema, escritos en este período se puede citar: A escola como organizaçáo complexa; Relaçóes de Poder na Escola; Delinqüéncia Académica. 
transformación de las relaciones en la escuela, en el sector empresarial las formas de gerenciar y controlar la mano de obra se van adaptando a las exigencias producidas por el mercado y por la presión de los trabajadores.

Los nuevos aires en relación con la organización del trabajo vienen del Japón, del milagro japonés, y las nuevas palabras de orden son: flexibilidad, calidad y evaluación. Las expectativas del capital en relación con el trabajador pasan a ser por un trabajador más autónomo, capaz de cambiar de tarea rápidamente, que posea una formación general y capacidad para adquirir nuevos conocimientos. Que sea creativo, capaz de tomar decisiones, como si el problema estuviera en el perfil del trabajador y no en la grave reducción de empleos existente.

Entre las primeras "herramientas" de esa nueva forma de gerenciar las empresas, adoptadas en el Brasil están los Círculos de Control de Calidad y el Just-in-Time. Tales novedades no encuentran desarmado a nuestro experto investigador: es de los primeros en denunciar las nuevas formas de control del trabajador. De la Faculdad de Educación de la Unicamp parte para el Japón, Eneida Ota Shiroma, quien, orientada por Tragtenberg ${ }^{4}$, debía ver de cerca en qué condiciones se producían esas nuevas técnicas de gestión en la Toyota.

Algunos pueden preguntarse por qué escogí este asunto para tratar aquí y creo que ahora, después de este largo viaje es posible explicar la importancia de haber estudiado los problemas de la administración desde el ángulo del poder. En verdad, estoy queriendo tratar de la universidad hoy y de la nueva moda que invade la política de enseñanza superior del Ministerio de Educación y Cultura y la política académica de buena parte de las universidades de América Latina. Quiero analizar cómo los principios de organización y gestión de las empresas llegaron a la reforma del Estado. La actual reforma del Estado y su consecuente política universitaria ha dado mucho qué hablar y más todavía qué pensar.

Según Marilena Chauí,

la reforma tiene un presupuesto ideológico básico: el mercado es portador de racionalidad socio-política y agente principal del bienestar de la República. Este presupuesto lleva a colocar derechos sociales (como la salud, la educación y la cultura) en el sector de servicios definidos por el mercado. De esta manera, la reforma recorta el espacio público democrático de los derechos y amplía el espacio privado, no sólo allí donde sería previsible —en las actividades ligadas a la producción económica - sino también donde no es admisible: en el campo de los derechos sociales conquistados.

La posición de la universidad en el sector de prestación de servicios confiere un sentido bastante determinado a la idea de autonomía universitaria e introduce términos como "calidad universitaria", "evaluación universitaria" y "flexibilización de la universidad" 5.

\footnotetext{
${ }^{4}$ Shiroma, Eneida Oto, Mudança tecnológica, qualificaçao e política de gestao, Campinas, UNICAMP, tesis de doctorado, 1994; Edmundo Escriváo Filho, CCQ e just-in-time: Uma análise integrada, Sáo Paulo, PUC/SP, 1992.

${ }^{5}$ Chauí, Marilena, "A universidade operacional”, Caderno Mais, Folha de Sao Paulo, p.3, 9/5/ 1999. Artículo traducido para este Dossier 
Estos tres términos que permean la política universitaria ya están en la práctica de las empresas privadas por lo menos hace dos décadas. Son elementos de la llamada "reingeniería empresarial" o de las nuevas formas de gestión, inspiradas, como vimos en el taylorismo. Son respuestas al proceso de globalización de la economía y a los rápidos cambios en el campo de las comunicaciones y de la informática. Hacen parte de las demandas de un mercado cada vez más internacionalizado.

Junto a los términos calidad, flexibilización y evaluación aparece otro: autonomía. Se espera que el trabajador sea autónomo, tenga auto-control y se conduzca según su autoevaluación. Se descarta o se desvalorizan las supervisiones y las direcciones. El trabajador debe, él mismo, gerenciar su desempeño, procurando la calidad de su trabajo.

Si en la gestión tradicional (taylorista) el trabajador era vigilado, controlado, regulado por los jefes, este proceso se restringía al tiempo de trabajo estipulado o contratado. En la gestión por evaluación, todos los espacios de la vida del trabajador están vigilados, pues, el vigía, que es él mismo, jamás se abandona. Mercado y concurrencia son las dos caras de esta moneda que permanece en la sombra de la evaluación y que aparece como instrumento para garantizar la calidad y la flexibilización.

Volvamos pues, al problema de la universidad que, como institución social que es, no está al margen de este proceso, generado por las nuevas formas de gestión y de relaciones de trabajo. Esta institución atacada y defendida en diferentes momentos históricos, se expandió y consolidó teniendo como referencia el reconocimiento público de su legitimidad y de sus atribuciones ${ }^{6}$.

La historia reciente de la universidad brasilera, testimonia la violación de ese reconocimiento y legitimidad, cuando el gobierno militar, en la década del 60, le impone una reforma de antemano establecida por la lógica del mercado, según la cual, la función de la universidad pasa a no ser más la formación, la reflexión, la creación y la crítica, sino la formación rápida de profesionales requeridos como mano de obra altamente calificada por el mercado de trabajo. Esta reforma impuso cambios curriculares, estructurales, dio énfasis a los contenidos técnicos, restringió el espacio de las humanidades, en fin, preparó el terreno para los cambios que se verifican en los años 90 .

La implementación de una nueva fase de la política académica basada en el mercado comienza a aparecer nítidamente cuando los organismos gubernamentales, las instituciones de fomento a la investigación desarrollan una serie de criterios para evaluar el desempeño de las universidades, de los investigadores-docentes y de los alumnos. La idea de evaluar las instituciones públicas, especialmente las universidades, que habian conseguido autonomía en varios aspectos, con criterios elaborados fuera de ellas, expresa uno de dos presupuestos: o se considera la evaluación un problema simplemente técnico (léase "neutral") o se niega la autonomía universitaria y se declara, de fuera hacia dentro, los objetivos de la academia.

Aquí cabe considerar los diferentes agentes sociales involucrados en la trama política. Como bien lo denunciaron Chauí y Roberto Romano ${ }^{7}$, a propósito de la política oficial para

\footnotetext{
${ }_{7}^{6}$ Ibid., p. 3.

${ }^{7}$ Chauí, Op. cit. y Romano, Roberto, "Entrevista Explosiva", in Caros Amigos, no. 23, fevereiro de 1999.

Digitalizado por RED ACADEMICA
} 
las universidades brasileras, el Ministerio de Educación y sus asesores se encuentran, sin duda, interesados en adaptar el Estado a las demandas del modelo capitalista, procuran convertir la universidad brasilera, de una institución social comprometida en reflexionar sobre la sociedad y la historia, en una organización social productiva. Para entender la posición del Ministerio de Educación, que lidera la gestión por la evaluación desde el Estado, tomo una vez más las palabras de Chauí:

la calidad es definida como competencia y excelencia, cuyo criterio es la atención a las necesidades de modernización de la economía y desarrollo social y es medida por la productividad, orientada por tres criterios: cuánto produce una universidad, en cuánto tiempo y a qué costo lo produce. En otras palabras, los criterios de productividad: cantidad, tiempo y costo, son los que definirán los contratos de gestión ${ }^{8}$.

Esta política viene siendo puesta en práctica por todas partes: la atribución de becas de estudio por el CAPES [Centro de aperfeiçoamento do pessoal do ensino superior y la CNPq [Conselho Nacional de Pesquisa] tienen como criterio número uno la productividad del programa de posgrado, medida por la ecuación: número de alumnos por el número de profesores en años de curso. En la misma lógica se mide el desempeño de los profesores: número de páginas escritas en artículos o libros por tiempo de trabajo o, mejor, número de horas en sala de clase, por número de alumnos en curso, por tiempo de trabajo.

Lo que está siendo escrito, pensado o investigado, poco importa; sin embargo, importa mucho cuánto está siendo producido y dónde. Esta lógica de la productividad califica y descalifica programas, universidades y docentes. Pero lo que me parece más grave es el tipo de comportamiento que esta política ha generado en los medios académicos. Jóvenes investigadores, recién llegados a la universidad, presionados por tal política cuantitativa y evaluadora, están muy preocupados por una producción individual, por el artículo que necesitan escribir y dónde publicarlo; quieren saber cuál revista está más reputada o tiene más valor en las evaluaciones de CAPES/CNPq. Construir un espacio de investigación con colegas y alumnos, donde se discuta la relevancia social de los temas, la coherencia y el significado de ciertos enfoques escapa a la mirada de estos investigadores.

Las mismas instituciones de fomento a la investigación, que implementan tal política creadora de competencia, construyen el discurso de la naturaleza interdependiente e interdisciplinar de la investigación. Mantienen proyectos de incentivo a la formación de grupos de excelencia pero preveen la retribución individual por el desempeño. De esta manera, los grupos de excelencia se convierten en espacios de lucha y competencia.

Se me ocurre preguntar lo que habría sucedido con la producción intelectual de Tragtenberg si en los años 80 él hubiese sido alcanzado por tal lógica política. ¿Qué habría sucedido con sus más de 200 artículos publicados en la columna "No batente", del diario Noticias Populares? Es evidente que publicar una columna semanal en Noticias Populares no tiene valor alguno para CAPES y CNPq, no cualifica el programa de posgrado en el que actúa este intelectual. Sin embargo, Mauricio, sabía claramente para quién quería escribir, con quién tenía interés en dialogar, quién lee-ría esta columna. Él, sin duda, usó otro criterio al optar por esa forma de trabajo. Ponderó la relevancia social y política de este diálogo. Su horizonte era la sociedad y no sólo su propia carrera académica.

\footnotetext{
${ }^{8}$ Chauí. p.3.

Digitalizado por RED ACADEMICA
} 
Pero yo hablaba de observar los diferentes agentes sociales involucrados en esta historia. Vimos la posición del Ministerio y de las instituciones de fomento. Cabe ahora observar lo que ocurre en el ámbito de las universidades, en las unidades de enseñanza e investigación, no sólo cómo esa política nos viene afectando a cada uno, sino a las universidades, facultades y centros de investigación.

Veamos, pues, algunos hechos puntuales que acaban por revelar el pensamiento vigente. En los últimos años, las distintas universidades del país están abriendo muchos cursos de posgrado; basta con conseguir el reconocimiento de la institución (o mejor, el título de universidad) para que ya sus administradores piensen en abrir un programa de posgrado. Entre las áreas de mayor oferta está la de educación, lo cual es comprensible pues las universidades y las instituciones de enseñanza superior están obligadas a titular a sus profesores según los índices considerados aceptables por los patrones de la CAPES/CNPq.

Considerando que un programa de posgrado en educación se propone formar docentes de enseñanza superior y que está abierto a cualquier área del conocimiento, nada más económico que abrir programas de posgrado en educación, garantizando que en primera instancia se titule al personal de planta. Este asunto -movido por la lógica económicasería grave si sólo se desvirtuaran los objetivos de un programa de posgrado en educación; pero, en verdad, se hace más que eso: se desvirtúan los objetivos propios de todo programa de esta naturaleza, cuales son la investigación y la producción de conocimiento. En la forma como ellos son estructurados en la actualidad, son simplemente distribuidores de credenciales para los que ya son profesores universitarios. Como diría Mauricio, se distribuyen diplomas legitimadores de una función burocrática. No preciso hablar de las presiones salariales sobre los profesores de las universidades públicas, que han funcionado como mecanismo generador de colaboración entre los profesores titulados de las instituciones públicas y esos nuevos programas de posgrado.

Esta política que vuelve la formación académica una formación instrumental parece no tener límite. Están siendo creados, por toda América Latina, cursos de posgrado en Evaluación Institucional y Aprendizaje. No sólo en nuevas instituciones de enseñanza superior, sin tradición o reconocimiento, sino también en instituciones públicas de prestigio. Intento observar estos proyectos haciéndome las preguntas ausentes en la política actual: ¿Qué significa la formación que está siendo propuesta en estos programas? ¿A quién sirven? ¿Para qué?

Elegir la evaluación como tema de un posgrado, con maestría y doctorado, significa atribuir relevancia y legitimidad al tema, colocándolo en condiciones de equivalencia en relación con maestrías y doctorados en historia, filosofía política, sociología o literatura, sólo para tomar algunos ejemplos. Esta idea me ha proporcionado cierta incomodidad y me hace pensar en las estrategias usadas por diferentes agentes sociales para constituir un campo de saber y legitimarlo. Así, me pongo a pensar sobre las orígenes de la evaluación, en qué momentos y en qué espacios gana importancia, al punto de sustantivarse, ganar cuerno, volverse objeto.

Evaluar: verbo, acción. Evaluar: algo que se realiza sobre alguna cosa existente (real). Evaluar la enseñanza, evaluar los profesores, evaluar la universidad. Pero evaluar, ¿en relación con qué? ¿A los objetivos fijados, a un patrón establecido, a las metas organizacionales? En fin, siempre en relación con. Así, evaluar remite a otros dos temas: planear, juzgar. 
Si asociamos evaluación con planeación, caemos en el universo de la administración ya discutido aquí. Sin embargo, nunca está de más recordar que en la administración tradicional, quien planea y evalúa no es quien desarrolla el trabajo, sino quien administra. En las nuevas formas de gestión, quien evalúa es el propio ejecutor, pero quien establece los patrones en relación con qué se evalúa es el mercado. Por tanto, en los dos casos, la evaluación funciona como mecanismo de opresión sobre quien trabaja.

Asociar "evaluación" con 'juicio", implica necesariamente la idea de clasificación, tema además estudiado ampliamente por Foucault, Apple y Bourdieu, entre otros. Me gustaría retomar un fragmento del análisis hecho por Elías Canetti sobre los actos de sentenciar y juzgar:

El hombre tiene una profunda necesidad de volver siempre a clasificar todas las personas que pueda imaginar. Dividiendo el número vago y amorfo de los existentes en dos grupos y colocándolos uno delante del otro, les atribuye algo semejante a "densidad". Los concentra como si tuvieran que luchar entre sí; los radicaliza y los llena de enemistad. De la manera como los imagina, de la manera como los quiere, ellos tienen que quedar necesariamente unos contra los otros. La separación entre "bueno" y "malo" es un método antiquísimo de clasificación dualista, que sin embargo nunca es enteramente conceptual ni totalmente pacífica. Ella implica la existencia de tensión entre los dos lados y es importante que la sentencia cree y renueve esta tensión ${ }^{9}$.

Releyendo este texto de Canetti, veo claramente los efectos de la evaluación (juicio) de la CAPES sobre los programas de posgrado, clasificándolos, colocando unos contra otros, definiendo los que deben ser apoyados y los que deben ser castigados, por ejemplo con suspensión de becas. En una evaluación clasificatoria, en la cual son atribuidas notas, no hay espacio para pensar las diferencias, debatir los enfoques. Existe un patrón establecido por el evaluador.

Pero la pregunta se mantiene: ¿será posible atribuir relevancia a la investigación y formación en evaluación, sea ella institucional o del aprendizaje, hasta el punto de justificar la realización de programas de posgrado? La creación de espacios en las facultades de educación, para este tipo de programas que forma posibles cuadros para la gestión evaluativa, ¿no estará contribuyendo a titular personas que, con la legitimidad del título, se abrogarán el derecho a definir los patrones de excelencia para la universidad?

Pienso que el tema de la administración aquí abordado, que de mero ejercicio de poder gana legitimidad científica y se establece como campo de saber, ya sería suficiente para que por lo menos no se acepte pasivamente este nuevo paquete con sellos internacionales del tipo Fundación Ford, Banco Mundial...

De mi parte, prefiero continuar confiando en la comunidad académica en su conjunto. No veo la evaluación como un problema técnico, sino como un problema político. Si los jueces para saber juzgar deben estudiar: derecho, filosofía, historia, o sea, deben conocer a fondo lo que se está juzgando -el ser humano y la sociedad-, me parece prudente que sean llamados a ocuparse de este asunto las universidades, los docentes investigadores y los estudiantes, aquellos que conocen mejor la institución, su historia y su lugar social.

\footnotetext{
${ }^{9}$ Canetti, Elias, Massa e Poder, Sáo Paulo, Brasilia, Melhoramentos, Editora de la UNB, 1983, p.331. 